Correspondence

Junyan Liu

liujy2005@yahoo.com.cn

Received 18 June 2008

Accepted 5 December 2008

\section{Enhanced immune response and protection efficacy of a DNA vaccine constructed by linkage of the Mycobacterium tuberculosis Ag85B-encoding gene with the BVP22-encoding gene}

\author{
Wanhong Yao, ${ }^{1}$ Shengwu Liu, ${ }^{2}$ Xueju Qu, ${ }^{2}$ Shaobo Xiao, ${ }^{3,4}$ Yan Liu ${ }^{2}$ \\ and Junyan Liur ${ }^{2,5}$
}

\author{
${ }^{1}$ Department of Microbiology and Parasitology, School of Medicine, Wuhan University, Wuhan, \\ PR China \\ ${ }^{2}$ Department of Immunology, School of Medicine, Wuhan University, Wuhan, PR China \\ ${ }^{3}$ Laboratory of Animal Virology, College of Veterinary Medicine, Huazhong Agricultural University, \\ Wuhan 430071, Hubei, PR China \\ ${ }^{4}$ National Key Laboratory of Agricultural Microbiology, Huazhong Agricultural University, Wuhan \\ 430071, Hubei, PR China \\ ${ }^{5}$ Animal Center of Wuhan University, Wuhan University, Wuhan, PR China
}

\begin{abstract}
Plasmid DNA vaccines have been widely explored for use in tuberculosis immunization but their immunogenicity needs improvement. In the present study, we incorporated the bovine herpesvirus 1 VP22 (BVP22)-encoding gene, which encodes a protein that demonstrates a capability for disseminating the expressed antigen to neighbouring cells, into a DNA vector in which it was fused to the Ag85B-encoding gene of Mycobacterium tuberculosis (Mtb), and investigated whether this linkage could enhance immune response and protective efficacy in $\mathrm{C} 57 \mathrm{BL} / 6$ mice compared to plasmid DNA encoding Ag85B alone. After immunization in mice, Ag85B-specific ELISA antibodies and spleen lymphocyte proliferative responses induced by DNA co-expressing BVP22 and Ag85B were significantly higher than those obtained in mice immunized with Ag85Bencoding DNA alone, except for the number of gamma interferon secreting cells. In addition, based on histopathological examination and bacterial-load determination in lung and spleen, protection against intravenous Mtb H37Rv challenge evoked by the BVP22-Ag85B DNA immunization exceeded the response elicited by Ag85B DNA alone, which was not significantly different from that provided by Bacillus Calmette-Guérin (BCG). These results suggested that DNA vaccine consisting of BVP22 and Ag85B-encoding DNA enhanced immune response and protection against intravenous Mtb H37Rv challenge in mice, indicating that BVP22-encoding DNA might be a promising tool to enhance TB DNA vaccine efficacy.
\end{abstract}

\section{INTRODUCTION}

Despite the widespread use of Bacillus Calmette-Guérin (BCG) vaccine and effective drug therapy, tuberculosis (TB) still remains a major global health threat. The incidence of TB has dramatically increased worldwide in the past few decades by the synergy of HIV pandemic, the increasingly drug-resistant $\mathrm{TB}$, e.g. multidrug-resistant and even extensively drug-resistant isolates, and the large scale mobility of the world population (Andersen, 2007; Reece \&

Abbreviations: BCG, Bacillus Calmette-Guérin; HE, haematoxylin and eosin; IFN- $\gamma$, gamma interferon; Mtb, Mycobacterium tuberculosis; MTT, 3-(4,5-dimethylthiazol-2-yl)-2,5-diphenyltetrazolium bromide; TB, tuberculosis; WHO, World Health Organization.
Kaufmann, 2008). The currently available TB vaccine, BCG, has some undesirable drawbacks. Among them are an inefficiency in preventing adult pulmonary TB, lack of safety in immunocompromised individuals, interference with tuberculin skin test screening and considerably variable efficacy (Hesseling et al., 2003, 2007; Andersen \& Doherty, 2005; Gupta et al., 2007; WHO, 2007). Consequently, the development of safer and more efficient vaccines, as an alternative or complement to BCG, is urgently needed to facilitate worldwide control or eradication of TB in the future (Kaufmann, 2007; Young \& Dye, 2006).

Among the novel vaccine candidates, plasmid DNA-based $\mathrm{TB}$ vaccines have drawn close attention because of their 
unique features compared to conventional live or subunit vaccines. The potency of plasmid DNAs expressing a variety of immunogenic Mycobacterium tuberculosis (Mtb) antigens has been intensively evaluated. Unfortunately, their performance is generally not superior to BCG, especially in large animals. However, the recent licensure of a DNA vaccine in horses highlights the potential of DNA vaccine technology in the prevention of $\mathrm{TB}$ infection (Ulmer et al., 2006). Besides, it is generally believed that novel TB vaccines will be tested in the context of the widely used BCG and perhaps different kinds of vaccines are needed for the eradication of TB (Sander \& McShane, 2007; Mitsuyama \& McMurray, 2007). As a result, enhancement of TB DNA vaccine efficacy has become the active field of current research.

It is well documented that VP22, a tegument protein of some alphaherpes viruses, has a unique property of being involved in intercellular transport and can be applied to improve DNA vaccine efficacy, despite uncertainty over the mechanism of action (Elliott \& O'Hare, 1997; Phelan et al., 1998; Oliveira et al., 2001; Hung et al., 2001, 2002; Michel et al., 2002; Zavaglia, et al., 2003; Qiu, et al., 2004; Kim et al., 2004; Zheng et al., 2005, 2006; Lemken et al., 2007b). For this reason, bovine herpesvirus 1 VP22 (BVP22)encoding DNA was chosen in the current study to test whether a fusion DNA vaccine created by the linkage of BVP22-encoding DNA to Mtb Ag85B-encoding DNA would induce an enhanced immune response and protection in C57BL/C mice compared to DNA encoding Ag85B alone. Another reason for selecting BVP22 was that it is the most efficient intercellular spreading motif identified according to the data available so far (Harms et al., 2000). Ag85B is a well-known and well-characterized protein of $\mathrm{Mtb}$, and plasmid DNA encoding Ag85B has shown partial protection in mice as well as in guinea pigs (Teixeira et al., 2006; Ko et al., 2005; Kamath et al., 1999; Lozes et al., 1997; Ulmer et al., 1997).

\section{METHODS}

Plasmid construction. The gene encoding Ag85B was amplified from Mtb H37Rv genomic DNA with a forward primer, 5'TTTGGATCCGGCATGTTCTCCCGGCCGGGGCTG-3', containing a BamHI site and an initiation codon, and a reverse primer, $5^{\prime}-$ GATGAATTCAGCCGGCGCCTAACG-3', containing an EcoRI site and a termination codon. The resulting PCR product was ligated to pMD18-T vector (TaKaRa Bio) and then sequenced. The BVP22 sequence without a stop codon was isolated from pMD-BVP22 (kindly provided by Professor Xiao, Huazhong Agricultural University) by digestion with BglII and BamHI. The target genes encoding Ag85B and BVP22 were subsequently cloned into the eukaryotic expression plasmid pcDNA3.1 $(+)$ (Invitrogen), and the new plasmids were named pcAg85B and pcBVP22, respectively. For construction of pcBVP22-Ag85B, the BVP22 sequence was inserted to pcAg85B at the BamHI site, which is on the upstream of Ag85B sequence. These recombinant plasmids were confirmed by sequence analysis. The pcDNA3.1 $(+)$ plasmid was used as the negative control. All plasmids for transfection and animal immunization were prepared and purified using the Wizard Plus maxiprep purification system (Promega).
Expression and purification of recombinant protein in $E$. coli. Expression of recombinant Ag85B protein in E. coli BL21 (DE3) was performed using the prokaryotic expression plasmid pET-28a, which contains the same insert as in pcAg85B and a $6 \times$ His tag at the $\mathrm{N}$ terminus to facilitate purification through $\mathrm{Ni}^{2+}$-charged column chromatography (Qiagen). Fractions were examined on an SDS-12\% polyacrylamide gel and recombinant protein detected by Western blot analysis using antisera from active TB patients. The protein was further purified according to the manufacturer's instructions and used as an antigen for identifying specific ELISA antibodies.

Transfection and detection of antigen expression. To evaluate in vitro expression of $\mathrm{Ag} 85 \mathrm{~B}$ and the fusion protein BVP22-Ag85B, HeLa cells [China Center of Type Culture Collection (CCTCC)] were transfected with the constructed plasmid DNAs using Lipofectamine 2000 reagent (Invitrogen) as described previously (Ju et al., 2005). Cells were harvested $48 \mathrm{~h}$ after transfection and transient expression was detected by Western blot, using anti-recombinant-Ag85B antiserum developed in mice. The specificity of the antiserum had been tested previously using ELISA.

Immunization of mice and challenge. A total of 90 female C57BL/ 6 mice were randomly assigned to 6 groups of 15 mice each and immunized with plasmid constructs. These mice were obtained from the Animal Center of Wuhan University at specified-pathogen free grade, body weight 16-18 g, aged 4-6 weeks at the beginning of the experiment, and housed in the Animal Facility of Wuhan University with autoclaved food and water ad libitum. Infection studies were performed in a biosafety level 3 facility. The mice were inoculated intramuscularly with $50 \mu \mathrm{l} 0.5 \%$ bupivacaine hydrochloride (Sigma) into the quadriceps muscle of each hind leg, 5 days before immunization, followed by immunization with $100 \mu$ l sterile PBS with or without $100 \mu \mathrm{g}$ plasmid construct intramuscularly three times at 2-week intervals. Six weeks after the last immunization, five mice from each group were sacrificed to determine the immunogenicity of the vaccines and controls at the time of challenge. The remaining ten mice per group were challenged intravenously in a lateral tail vein with $0.1 \mathrm{ml}\left(10^{6}\right.$ bacilli) suspension of $\mathrm{Mtb} \mathrm{H} 37 \mathrm{Rv}$ per animal. Five weeks after challenge, all mice were sacrificed for assessment of bacterial growth and histopathological analysis as described previously (Liu et al., 2006). Negative controls were mice immunized with $100 \mu \mathrm{l}$ PBS, pcDNA3.1(+) or pBVP22; the positive controls were mice inoculated with a single dose of BCG subcutaneously $\left(10^{6}\right.$ c.f.u. per animal) at the time of the first prime dose of candidate DNA vaccine.

Analysis of Ag85B-specific antibody responses by ELISA. To evaluate anti-Ag85B humoral responses, sera were collected from five immunized mice in each group 6 weeks after the last immunization, and levels of anti-Ag85B total IgG antibody in serum samples were determined by ELISA. Briefly, each well was coated with $100 \mu \mathrm{l}$ purified recombinant $85 \mathrm{~B}$ protein $\left(8 \mu \mathrm{g} \mathrm{ml}^{-1}\right)$ overnight at $4{ }^{\circ} \mathrm{C}$ and incubated with $100 \mu \mathrm{l}$ serial twofold dilutions of serum for $60 \mathrm{~min}$ at $37{ }^{\circ} \mathrm{C}$, before the addition of $100 \mu \mathrm{l} \mathrm{HRP-conjugated} \mathrm{goat} \mathrm{anti-}$ murine $\operatorname{IgG}(1: 2000)$ and substrate solution TMB. Each sample was tested in triplicate. The reaction was stopped by addition of $2 \mathrm{M}$ sulfuric acid and the absorbance was measured at $490 \mathrm{~nm}$ using a microplate reader (Tecan). Antibody titres were calculated as the highest dilution that gave a positive reading. The cut-off value was set as twice the mean absorbency of sera from the negative control.

Spleen lymphocyte proliferation assay. Spleen lymphocyte proliferation response was measured by the 3-(4,5-dimethylthiazol2-yl)-2,5-diphenyltetrazolium bromide (MTT) colorimetric method. Briefly, splenocytes were prepared from five mice of each group and lymphocytes were isolated by density-gradient centrifugation on

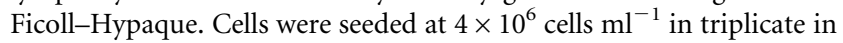


96-well plates, and cultured in RPMI 1640 medium with $10 \%$ heatinactivated fetal bovine serum (FBS), $100 \mathrm{U}$ penicillin $\mathrm{ml}^{-1}, 100 \mu \mathrm{g}$ streptomycin $\mathrm{ml}^{-1}$ and $2 \mathrm{mM}$ L-glutamine at $37{ }^{\circ} \mathrm{C}$ and $5 \% \mathrm{CO}_{2}$ with or without Mtb antigen stimulation. After $68 \mathrm{~h}$ incubation, $10 \mu \mathrm{l}$ MTT (Sigma) was added to each well and plates were incubated for a further $4 \mathrm{~h}$. At the end of the incubation, the supernatants in each well were gently removed after the plates were centrifuged at 1000 r.p.m. (374 $\mathrm{g}$ ) in a Heraeus Biofuge Stratos for $8 \mathrm{~min} ; 150 \mu \mathrm{l}$ DMSO were then added into each well to solubilize the formazan precipitates for $10 \mathrm{~min}$ under oscillation. The cells containing RPMI 1640/FBS only were used as a negative control and the cells stimulated with concanavalin A $\left(5 \mu \mathrm{g} \mathrm{ml}^{-1}\right.$; Sigma) were used as a positive control. The stimulation index was calculated as the ratio of $A_{570}$ of stimulated to that of unstimulated proliferation. The recombinant protein Ag85B was used as the antigen in this study at a final concentration of $8 \mu \mathrm{g} \mathrm{ml}^{-1}$.

Gamma interferon (IFN- $\gamma$ ) ELISPOT assay. IFN- $\gamma$-producing cells were quantified using a cytokine-specific enzyme-linked immunospot assay (ELISPOT) kit (U-Cytech) according to the manufacturer's instructions. Briefly, 96-well nitrocellulose-backed plates were coated with $100 \mu \mathrm{l}$ anti-mouse IFN- $\gamma$ mAb overnight at $4{ }^{\circ} \mathrm{C}$, then the wells were washed with PBS ( $\mathrm{pH} 7.4$ ) and blocked for $2 \mathrm{~h}$ with $5 \%$ BSA. Lymphocytes isolated from the mouse spleen following treatment were added to the wells $\left(2 \times 10^{5}\right.$ per well $)$ and cultured for $18 \mathrm{~h}$ in RPMI 1640 alone (negative control) or with $8 \mu \mathrm{g} \mathrm{ml}^{-1}$ recombinant Ag85B or $5 \mu \mathrm{g} \mathrm{ml}^{-1}$ concanavalin A (positive control) in triplicate. After five washes with PBS containing $0.05 \%$ Tween 20, $100 \mu \mathrm{l}$ biotinylated rabbit anti-IFN- $\gamma$ antibody solution [prepared according to the guidelines of the manufacturer (U-Cytech)] was added per well and incubation continued for $1 \mathrm{~h}$ at room temperature. Following washes with PBS containing $0.05 \%$ Tween 20, $50 \mu \mathrm{l} \varphi$-labelled antibiotin antibody solution [prepared according to the guidelines of the manufacturer (U-Cytech)] was added and incubated for $1 \mathrm{~h}$ at room temperature. After rewashing, $30 \mu \mathrm{l}$ freshly prepared activator I/II solution were added to each well and plates were incubated at room temperature in the dark. Spots representing individual cytokineproducing cells were visualized by developing with the substrate 3amino-9-ethylcarbazole and then counted using an immunospot image analyser.

Statistical analysis. Data were expressed as the means \pm SEM. Oneway ANOVA or Student's $t$-tests were performed using SPSS 11.5 software for comparison of results between different groups. $P$ values $<0.05$ were considered statistically significant.

\section{RESULTS AND DISCUSSION}

\section{Expression of Mtb Ag85B-encoding gene in HeLa cells}

The expression of Ag85B and fusion protein BVP22-Ag85B was examined by Western blot of the cell lysates following transfection of HeLa cells with pcAg85B and pcBVPAg85B. The study confirmed that cells transfected with these plasmids expressed the corresponding proteins, based on the anticipated size for Ag85B $(\sim 30 \mathrm{kDa})$ and BVP22Ag85B ( $65 \mathrm{kDa})$ (data not shown). In contrast, no band was observed in HeLa cells transfected with vector pcDNA3.1 or pcBVP22. These results verified the expression of the recombinant BVP22-Ag85B and Ag85B proteins in mammalian cells.

\section{Evaluation of IgG antibodies and spleen lymphocyte proliferation}

Levels of IgG antibodies and the lymphocyte proliferation induced by different vaccine schedules are shown in Figs 1 and 2. Both pcAg85B and pcBVP22-Ag85B elicited significantly higher levels of anti-Ag85B IgG antibodies and lymphocyte proliferation activities in mice $(P<0.05$ for both pcAg85B and pcBVP22-Ag85B immunized mice compared with the respective negative controls). Furthermore, pcBVP22-Ag85B vaccination significantly increased the levels of IgG antibodies and lymphocyte proliferation in mice compared with pcAg85B immunization $(P<0.05)$, and achieved a similar effect to BCG. No Ag85B-specific antibodies and proliferation response were detected in mice receiving PBS or control vector pcDNA3.1 or pcBVP22. These results demonstrated that immunization with DNA encoding BVP22-Ag85B fusion protein significantly enhanced anti-Ag85B specific IgG antibody levels and cell proliferation in mice.

\section{IFN- $\gamma$ production in response to $\mathbf{A g 8 5 B}$}

IFN- $\gamma$ production in response to recombinant Ag85B was measured in three mice of each group by ELISPOT before challenge. The ELISPOT assay revealed that mice vaccinated with pcAg85B, pcBVP22-Ag85B and BCG demonstrated higher levels of IFN- $\gamma$-producing cells in the spleen than did the PBS and vector controls. The highest number of IFN- $\gamma$-producing cells was detected in mice vaccinated with BCG, followed by pcBVP22-Ag85B and pcAg85B. In addition, the pcBVP22-Ag85B-vaccinated mice showed a

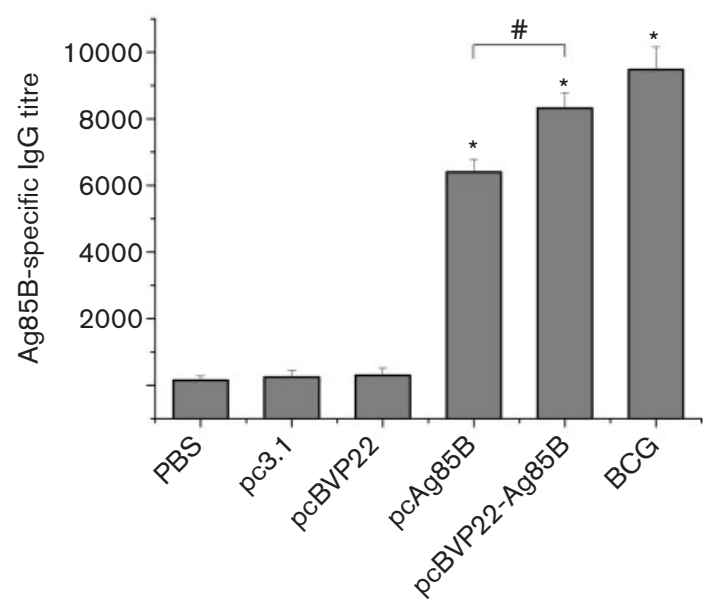

Fig. 1. Production of $\operatorname{lgG}$ against $A g 85 B$ protein in vaccinated mice. Mice were immunized with PBS, pcDNA3.1, pcBVP22, pcAg85B, pcBVP22-Ag85B or BCG. Sera were collected from five mice in each group 6 weeks after the last immunization. The total IgG titre against Ag85B protein was determined by ELISA. *, $P<0.05$ compared to the negative control groups (PBS, pcDNA3.1, or pcBVP22); \#, $P<0.05$ compared to the pcAg85B-immunized group. 


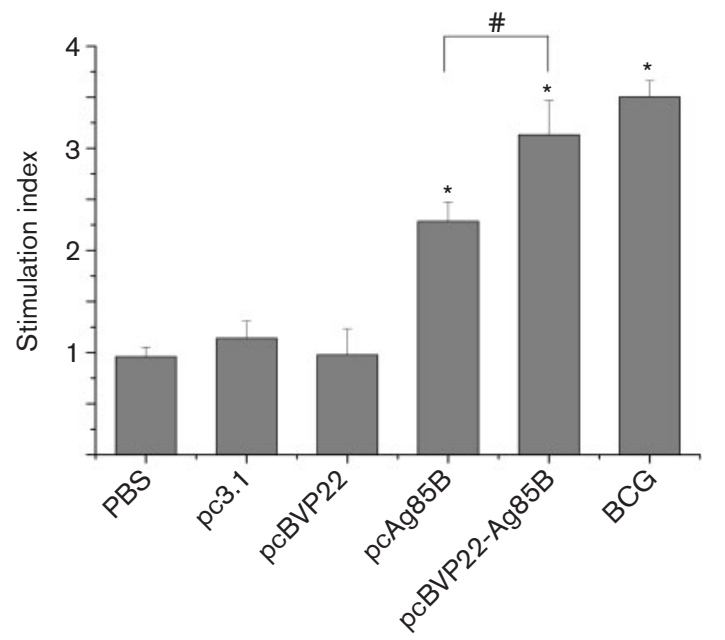

Fig. 2. Lymphocyte proliferative response after in vitro stimulation with recombinant Ag85B protein. Mice were immunized with PBS, pcDNA3.1, pcBVP22, pcAg85B, pcBVP22-Ag85B or BCG. Six weeks after the last immunization, mice were sacrificed, and spleen lymphocytes were isolated by density-gradient centrifugation on Ficoll-Hypaque and then stimulated with recombinant Ag85B proteins $\left(8 \mu \mathrm{g} \mathrm{ml}^{-1}\right)$ for $68 \mathrm{~h}$. Following incubation with MTT for $4 \mathrm{~h}$, the absorbance was determined in a microplate reader at 570 $\mathrm{nm}$. Samples were assayed in triplicate. Data represent the mean \pm SEM. ${ }^{*}, P<0.05$ compared to the negative control groups (PBS, pcDNA3.1, pcBVP22); \#, $P<0.05$ compared to the pcAg85B-immunized group.

prominent trend for elevated numbers of antigen specific IFN- $\gamma$-producing cells in the spleen in comparison to pcAg85B, even though it is not statistically significant (Fig. 3). In contrast, mice injected with pc3.1 or pcBVP22 did not show any increased IFN- $\gamma$-producing cell proliferation compared to PBS-immunized mice $(P>0.05)$. These data indicated that linkage of BVP22 to Ag85B enhanced the effect of the DNA vaccine on IFN- $\gamma$ producing $\mathrm{T}$ cells.

\section{c.f.u. determination}

Finally, we investigated whether the BVP22-Ag85B DNA vaccine enhanced protective efficacy against intravenous Mtb H37Rv infection in the C57BL/6 mouse model, based on the determination of bacterial load by c.f.u. counts in the lung and spleen at 5 weeks post-challenge. As shown in Table 1, immunization with either pcAg85B or pcBVP22Ag85B led to a significant reduction of mycobacterial loads in the lung or spleen in comparison to the PBS group $(P<0.05)$. More importantly, mice vaccinated with pcBVP22-Ag85B demonstrated a significant reduction of mycobacterial loads and dissemination compared to those vaccines with pcAg85B alone $(P<0.05)$. The effect of pcBVP22-Ag85B was not significantly different from that of BCG inoculation. No c.f.u. reduction was observed in the lung or spleen of mice vaccinated with either parental

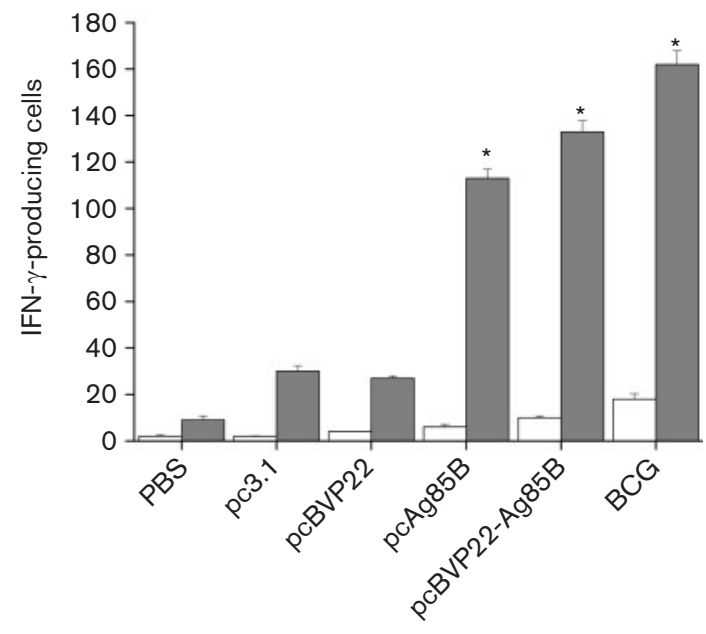

Fig. 3. Ag85B-specific IFN- $\gamma$-producing cells determined by ELISPOT assay. Mice were immunized with either PBS, or pcDNA3.1, pcBVP22, pcAg85B, pcBVP22-Ag85B or BCG. The ELISPOT assay was performed as described in Methods. Numbers represent mean numbers of spot-forming cells per $2 \times 10^{5}$ lymphocytes. *, $P<0.05$ compared to the respective negative control groups. White bars, no stimuli; grey bars, Ag85B.

vector pc3.1 or pcBVP22 compared to the PBS group. We have further analysed the relationship between the c.f.u. reduction and IFN- $\gamma$-producing response by Pearson correlation analysis and found that they are closely positively correlated ( $r=0.99, P<0.01$; data not shown). Along with the data presented in Fig. 3, these studies suggested that pcBVP22-Ag85B vaccine is superior to pcAg85B in both immunogenicity and protection efficacy, probably through amplification of IFN- $\gamma$-producing cells.

\section{Pathological changes in the lungs}

To assess the degree of lung lesions present following mycobacterial challenge in vaccinated mice, mouse lung tissue was fixed, sectioned and then stained with either Ziehl-Neelsen acid-fast reagent or haematoxylin and eosin (HE). As shown in Fig. 4(a), the Ziehl-Neelsen staining showed that mice immunized with pcAg85B, pcBVP22Ag85B and BCG were able to inhibit the growth of tubercle bacilli in the lung after the challenge. The lungs of mice from the PBS, pcDNA3.1 and pcBVP22 groups displayed a much higher density of acid-fast bacilli than those of mice vaccinated with pcAg85B, pcBVP22-Ag85B or BCG, consistent with the mycobacterial growth data presented above. In HE staining sections (Fig. 4b), lung tissue granulomatous lesions and inflammation changes were dramatically suppressed in mice vaccinated with pcAg85B, pcBVP22-Ag85B or BCG in comparison to the mice vaccinated with $\mathrm{PBS}$, pcDNA3.1 or pcBVP22, which showed a significant amount of consolidation, seroplastic infiltrates, necrosis and multiple coalescing granulomatous lesions in most of the lungs. The alveolar tissue from 
Table 1. Anti-TB protective immunity induced by immunization with DNA constructs

\begin{tabular}{|lcccc|}
\hline \multirow{2}{*}{ Group $\dagger$} & \multicolumn{3}{c|}{ Mean $\log _{\mathbf{1 0}}$ c.f.u. \pm SEM } \\
\cline { 2 - 5 } & Lung c.f.u. $\neq$ & Log c.f.u. reduction $\$$ & Spleen c.f.u. $\ddagger$ & Log c.f.u. reduction $\$$ \\
\hline PBS & $5.9443 \pm 0.0345$ & - & $6.0065 \pm 0.0482$ & - \\
pc3.1 & $5.9362 \pm 0.0192$ & 0.0081 & $6.0039 \pm 0.0191$ & 0.0026 \\
pcBVP22 & $5.9416 \pm 0.0312$ & 0.0027 & $5.9996 \pm 0.0218$ & 0.0069 \\
pc3.1-Ag85B & $5.3174 \pm 0.0276$ & $0.6269^{*}$ & $5.3328 \pm 0.1031$ & $0.6737^{\star}$ \\
pcBVP22-Ag85B & $4.9313 \pm 0.0592$ & $1.0130^{*}$ & $5.0031 \pm 0.0774$ & $1.0034^{*} \#$ \\
BCG & $4.7813 \pm 0.1129$ & $1.1630^{*}$ & $4.8669 \pm 0.1252$ & $1.1396^{*} \#$ \\
\hline
\end{tabular}

$\left\lceil\right.$ C57BL/6 mice were immunized with $10^{6}$ c.f.u. BCG subcutaneously once or with either $0.1 \mathrm{mg}$ plasmid DNA or an equal volume of PBS intramuscularly three times at 2-week intervals. Six weeks after the last DNA immunization, the mice were challenged with Mtb H37Rv intravenously.

$\$$ Number of bacteria isolated from the organ at 35 days after an intravenous challenge with $10^{6}$ c.f.u. of Mtb H37Rv per mouse ( $n=5$ ).

$\S$ The reduction in bacterial counts in the lungs of plasmid-vaccinated mice relative to the PBS-vaccinated mice; ${ }^{\star} P<0.05$, compared to respective negative controls; $\# P<0.05$, compared to pcAg85B-immunized group.
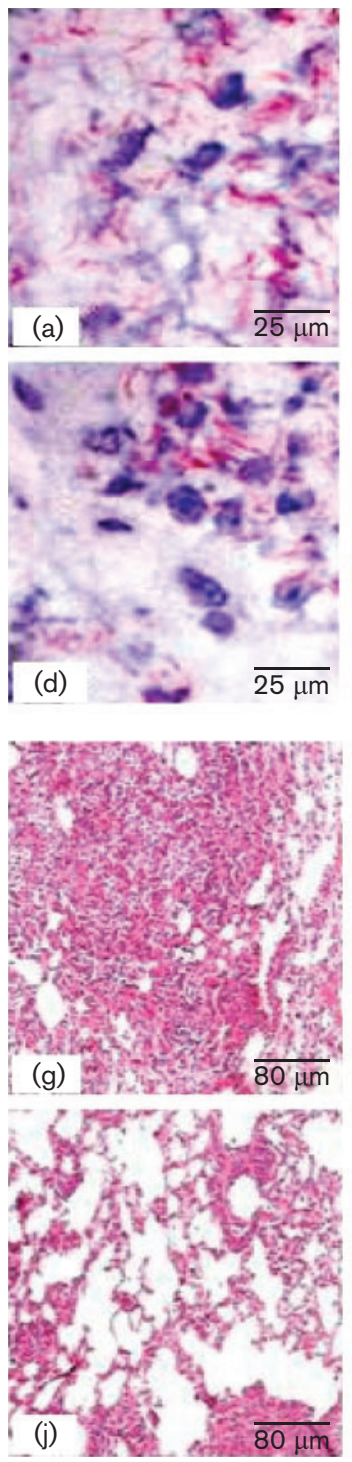

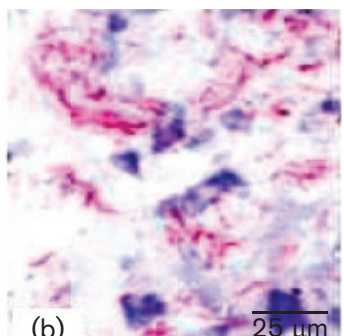

(b)
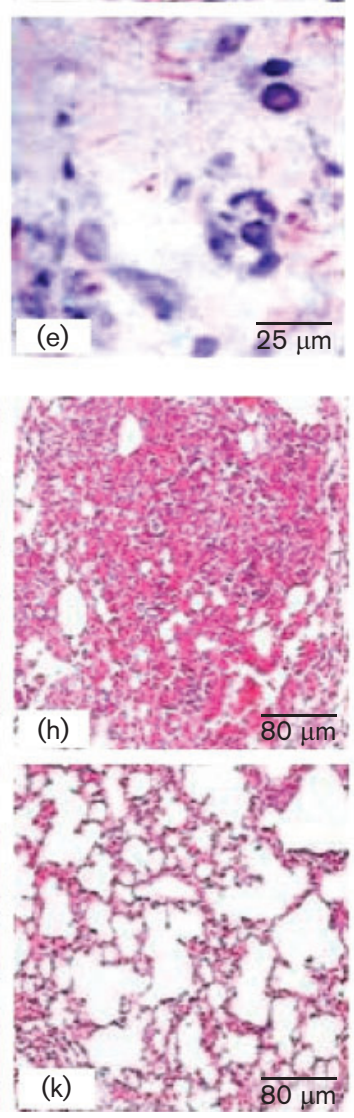
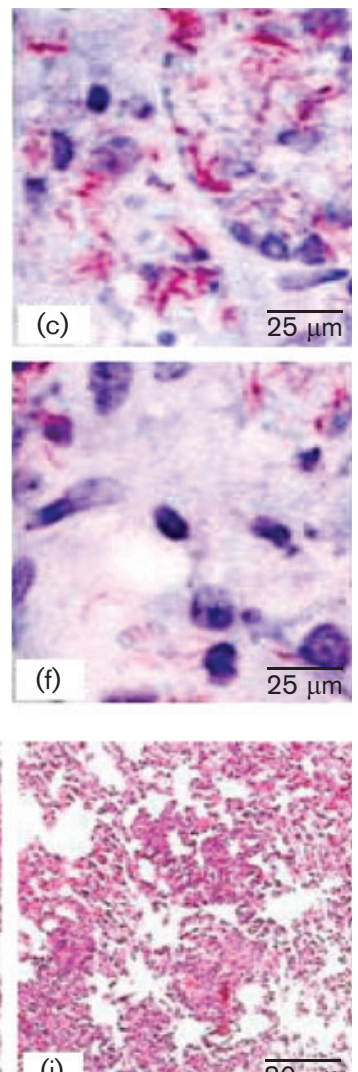

(i) 80 , $80 \mu \mathrm{m}$

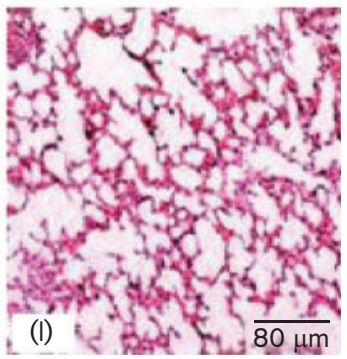

Fig. 4. Representative micrographs from acidfast bacilli staining ( $\times 1000$ magnification) $(a-f)$ and $\mathrm{HE}$ staining $(\times 100$ magnification) $(\mathrm{g}-\mathrm{l})$ of lung tissues from vaccinated mice challenged with $10^{6}$ c.f.u. Mtb H37Rv intravenously. Mice were immunized once with $10^{6}$ c.f.u. BCG or plasmid DNA as described in Methods. Six weeks after the last DNA immunization, mice were challenged with Mtb H37Rv intravenously. At 35 days following challenge, lung tissues from five mice in each group were isolated, fixed, and stained with Ziehl-Neelsen reagent $(a-f)$ and $\mathrm{HE}(g-l)$. $(a, g)$, mice injected with PBS; $(b, h)$, mice injected with pcDNA3.1; (c, i), mice injected with pcBVP22; $(d, j)$, mice vaccinated with pcAg85B; $(e, k)$, mice vaccinated with pcBVP22-Ag85B; $(f, l)$, mice vaccinated with $B C G$. 
BCG-vaccinated mice appeared to be intact, with very limited lung inflammation. In pcAg85B- and pcBVP22Ag85B-vaccinated mice, limited granulomatous lesions and slight inflammation were observed, especially in pcBVP22vaccinated mice. Taken together, these results suggest that pcAg85B, pcBVP22-Ag85B and BCG vaccination enhanced mouse immunity, improved protection against mycobacterial bacterial growth, and curtailed bacteria-induced inflammation.

Naked DNA vaccines have emerged as an attractive approach for novel TB vaccine development, and efforts have been made continuously to improve the immunogenicity of DNA vaccines by several strategies. VP22, a tegument protein of some alphaherpes viruses, has been reported to exhibit an unusual intercellular trafficking property of mediating protein spread from cell to cell, alone or as apart of fusion protein (Lemken et al., 2007a; Zheng et al., 2006; Mwangi et al., 2005; Michel et al., 2002; Elliott \& O'Hare, 1997). Recent studies have further demonstrated that DNA constructs that encode fusion proteins of VP22 linked to an antigen can increase the potency of DNA vaccines in inbred and outbred animals (Zheng et al., 2005; Saha, et al., 2006; Mwangi et al., 2007; Zakhartchouk et al., 2007). However, the capacity of VP22 to improve the immunogenicity and efficacy of TB DNA vaccines containing mycobacterial antigens remains to be elucidated. In our study, Ag85B, one of the most important immunogenic antigens of Mtb, was fused to BVP22 to investigate whether this linkage of BVP22 and Ag85B can improve the potency of Ag85B DNA vaccine. Our data clearly showed that vaccination with naked DNA encoding BVP22-Ag85B fusion protein significantly enhanced the Ag85B-specific Th1-type immune responses in C57BL/6 mice, as demonstrated by a significant enhancement of lymphocyte proliferation and specific antibody level compared to Ag85B DNA immunization. The intercellular trafficking property of VP22 might contribute to the enhanced immune responses, similar to results from previous studies in which VP22 was demonstrated to enhance the immunogenicity of various antigens fused to VP22. However, other mechanisms may play a role in the action of VP22 in the current study. Some researchers have found that VP22 contains T-cell epitopes (Braun et al., 2006). During immunization with the fusion protein, it is possible that the antigenic epitopes on VP22 stimulate lymphocytes and contribute to an inflammatory response that subsequently improves the antigen-specific response to antigen Ag85B. Clearly, the exact mechanism of action of VP22 remains to be elucidated. We believe that this can be achieved, at least partially, by evaluating the immune response in mice immunized with both pcBVP22 and pcAg85B plasmids, which is part of our future study.

Although mice immunized with pcBVP22-Ag85B did not show a significant difference in the numbers of IFN- $\gamma$ producing cells in comparison with those immunized with pcAg85B alone, they clearly showed enhanced protection because the bacterial load both in the lung and spleen were significantly reduced and lung pathology was prominently improved. We believe that this is probably due to an amplified immune response as the results of linear correlation analysis revealed that $\log$ c.f.u. reduction in the lung was closely correlated with IFN $-\gamma$ response.

In summary, our data have demonstrated that incorporation of the BVP22-encoding gene into a DNA vaccine encoding Mtb Ag85B enhanced the Ag85B-specific immune response and protection efficacy against intravenous Mtb $\mathrm{H} 37 \mathrm{Rv}$ challenge. Such an approach may be used to improve the potency of vaccines against $\mathrm{TB}$ in the future.

\section{REFERENCES}

Andersen, P. (2007). Tuberculosis vaccines - an update. Nat Rev Microbiol 5, 484-487.

Andersen, P. \& Doherty, T. M. (2005). The success and failure of BCG - implications for a novel tuberculosis vaccine. Nat Rev Microbiol 3, 656-662.

Braun, R. P., Payne, L. G. \& Dong, L. C. (2006). Characterization of the IFN- $\gamma$ T-cell responses to immediate early antigens in humans with genital herpes. Virol J 3, 54-68.

Elliott, G. \& O'Hare, P. (1997). Intercellular trafficking and protein delivery by a herpesvirus structural protein. Cell 88, 223-233.

Gupta, U. D., Katoch, V. M. \& McMurray, D. N. (2007). Current status of TB vaccines. Vaccine 25, 3742-3751.

Harms, J. S., Ren, X. D., Oliveira, S. C. \& Splitter, G. A. (2000). Distinctions between bovine herpesvirus 1 and herpes simplex virus type 1 VP22 tegument protein subcellular associations. J Virol 74, 3301-3312.

Hesseling, A. C., Schaaf, H. S., Hanekom, W. A., Beyers, N., Cotton, M. F., Gie, R. P., Marais, B. J., van Helden, P. \& Warren, R. M. (2003). Danish Bacille Calmette-Guérin vaccine-induced disease in human immunodeficiency virus-infected children. Clin Infect Dis 37, 12261233.

Hesseling, A. C., Marais, B. J., Gie, R. P., Schaaf, H. S., Fine, P. E. M., Godfrey-Faussett, P. \& Beyers, N. (2007). The risk of disseminated Bacille Calmette-Guérin (BCG) disease in HIV-infected children. Vaccine 25, 14-18.

Hung, C. F., Cheng, W. F., Chai, C. Y., Hsu, K. F., He, L. M., Ling, M. \& Wu, T. C. (2001). Improving vaccine potency through intercellular spreading and enhanced MHC class I presentation of antigen. J Immunol 166, 5733-5740.

Hung, C. F., He, L. M., Juang, J., Lin, T. J., Ling, M. \& Wu, T. C. (2002). Improving DNA vaccine potency by linking Marek's disease virus type 1 VP22 to an antigen. J Virol 76, 2676-2682.

Ju, W., Liu, J., Xiao, W., Liu, M. \& Qu, X. (2005). Construction of a eukaryotic expression system of HSP65 gene from Mycobacterium tuberculosis, and anti-HSP65 IgG produced in mice. J Med Microbiol 54, 3-6.

Kamath, A. T., Feng, C. G., MacDonald, M., Briscoe, H. \& Britton, W. J. (1999). Differential protective efficacy of DNA vaccines expressing secreted proteins of Mycobacterium tuberculosis. Infect Immun 67, 1702-1707.

Kaufmann, S. H. E. (2007). The contribution of immunology to the rational design of novel antibacterial vaccines. Nat Rev Microbiol 5, 491-504.

Kim, T. W., Hung, C.-F., Kim, J. W., Juang, J., Chen, P.-J., He, L. M., Boyd, D. A. K. \& Wu, T.-C. (2004). Vaccination with a DNA vaccine 
encoding herpes simplex virus type 1 VP22 linked to antigen generates long-term antigen-specific CD8-positive memory $\mathrm{T}$ cells and protective immunity. Hum Gene Ther 15, 167-177.

Ko, H. J., Ko, S. Y., Kim, Y. J., Lee, E. G., Cho, S. N. \& Kang, C. Y. (2005). Optimization of codon usage enhances the immunogenicity of a DNA vaccine encoding mycobacterial antigen Ag85B. Infect Immun 73, 5666-5674.

Lemken, M. L., Wolf, C., Wybranietz, W. A., Schmidt, U., Smirnow, I., Buhring, H. J., Mack, A. F., Lauer, U. M. \& Bitzer, M. (2007a). Evidence for intercellular trafficking of VP22 in living cells. Mol Ther 15, 310-319.

Lemken, M.-L., Graepler, F., Wolf, C., Wybranietz, W. A., Smirnow, I., Schmidt, U., Gregor, M., Bitzer, M. \& Lauer, U. M. (2007b). Fusion of HSV-1VP22 to a bifunctional chimeric SuperCD suicide gene compensates for low suicide gene transduction efficiencies. Int $J$ Oncol 30, 1153-1161.

Liu, B., Liu, S., Qu, X. \& Liu, J. (2006). Construction of a eukaryotic expression system for granulysin and its protective effect in mice infected with Mycobacterium tuberculosis. J Med Microbiol 55, 1389-1393.

Lozes, E., Huygen, K., Content, J., Denis, O., Montgomery, D. L., Yawman, A. M., Vandenbussche, P., Van Vooren, J. P., Drowart, A. \& other authors (1997). Immunogenicity and efficacy of a tuberculosis DNA vaccine encoding the components of the secreted antigen 85 complex. Vaccine 15, 830-833.

Michel, N., Osen, W., Gissmann, L., Schumacher, T. N. M., Zentgraf, H. \& Muller, M. (2002). Enhanced immunogenicity of HPV 16 E7 fusion proteins in DNA vaccination. Virology 294, 47-59.

Mitsuyama, M. \& McMurray, D. N. (2007). Tuberculosis: vaccine and drug development. Tuberculosis (Edinb) 87, S10-S13.

Mwangi, W., Brown, W. C., Splitter, G. A., Zhuang, Y., Kegerreis, K. \& Palmer, G. H. (2005). Enhancement of antigen acquisition by dendritic cells and MHC class II-restricted epitope presentation to CD $4^{+} \mathrm{T}$ cells using VP22 DNA vaccine vectors that promote intercellular spreading following initial transfection. J Leukoc Biol 78, 401-411.

Mwangi, W., Brown, W. C., Splitter, G. A., Davies, C. J., Howard, C. J., Hope, J. C., Aida, Y., Zhuang, Y., Hunter, B. J. \& Palmer, G. H. (2007). DNA vaccine construct incorporating intercellular trafficking and intracellular targeting motifs effectively primes and induces memory B- and T-cell responses in outbred animals. Clin Vaccine Immunol 14, 304-311.

Oliveira, S. C., Harms, J. S., Afonso, R. R. \& Splitter, G. A. (2001). A genetic immunization adjuvant system based on BVP22-antigen fusion. Hum Gene Ther 12, 1353-1359.

Phelan, A., Elliott, G. \& O'Hare, P. (1998). Intercellular delivery of functional p53 by the herpesvirus protein VP22. Nat Biotechnol 16, 440-443.
Qiu, Z. H., Harms, J. S., Zhu, J. \& Splitter, G. A. (2004). Bovine herpesvirus tegument protein VP22 enhances thymidine kinase/ ganciclovir suicide gene therapy for neuroblastomas compared to herpes simplex virus VP22. J Virol 78, 4224-4233.

Reece, S. T. \& Kaufmann, S. H. E. (2008). Rational design of vaccines against tuberculosis directed by basic immunology. Int $\mathrm{J}$ Med Microbiol 298, 143-150.

Saha, S., Yoshida, S., Ohba, K., Matsui, K., Matsuda, T., Takeshita, F., Umeda, K., Tamura, Y., Okuda, K. \& other authors (2006). A fused gene of nucleoprotein (NP) and herpes simplex virus genes (VP22) induces highly protective immunity against different subtypes of influenza virus. Virology 354, 48-57.

Sander, C. \& McShane, H. (2007). Translational mini-review series on vaccines: development and evaluation of improved vaccines against tuberculosis. Clin Exp Immunol 147, 401-411.

Teixeira, F. M., Teixeira, H. C., Ferreira, A. P., Rodrigues, M. F., Azevedo, V., Macedo, G. C. \& Oliveira, S. C. (2006). DNA vaccine using Mycobacterium bovis Ag85B antigen induces partial protection against experimental infection in BALB/c Mice. Clin Vaccine Immunol 13, 930-935.

Ulmer, J. B., Liu, M. A., Montgomery, D. L., Yawman, A. M., Deck, R. R., DeWitt, C. M., Content, J. \& Huygen, K. (1997). Expression and immunogenicity of Mycobacteriun tuberculosis antigen 85B by DNA vaccination. Vaccine 15, 792-794.

Ulmer, J. B., Wahren, B. \& Liu, M. A. (2006). Gene-based vaccines: recent technical and clinical advances. Trends Mol Med 12, 216-221.

WHO (2007). Global advisory committee on vaccine safety, 29-30 November 2006. Wkly Epidemiol Rec 82, 18-24.

Young, D. \& Dye, C. (2006). The development and impact of tuberculosis vaccines. Cell 124, 683-687.

Zakhartchouk, A. N., Viswanathan, S., Moshynskyy, I., Petric, M. \& Babiuk, L. A. (2007). Optimization of a DNA vaccine against SARS. DNA Cell Biol 26, 721-726.

Zavaglia, D., Favrot, M.-C., Eymin, B., Tenaud, C. \& Coll, J.-L. (2003). Intercellular trafficking and enhanced in vivo antitumour activity of a nonvirally delivered P27-VP22 fusion protein. Gene Ther 10, 314325.

Zheng, C. F., Babiuk, L. A. \& Littel-Van Den Hurk, S. V. (2005). Bovine herpesvirus 1 VP22 enhances the efficacy of a DNA vaccine in cattle. J Virol 79, 1948-1953.

Zheng, C. F., Brownlie, R., Huang, D. Y., Babiuk, L. A. \& van Drunen Littel-van den Hurk, S. (2006). Intercellular trafficking of the major tegument protein VP22 of bovine herpesvirus-1 and its application to improve a DNA vaccine. Arch Virol 151, 985-993. 